\title{
Coregistration of Small Animal PET and Autoradiography for in vivo-ex vivo Comparison
}

\author{
Soo-Min Song ${ }^{1}$, Min-Jeong Kim ${ }^{1}$, Joung-Min Lee ${ }^{1}$, Hye-Jin Park ${ }^{1}$, \\ KyeongMin $\mathrm{Kim}^{2}$, Gi-Jeong Cheon ${ }^{2}$, and Myoung-Hee Kim ${ }^{1,3, *}$ \\ ${ }^{1}$ Department of Computer Science and Engineering \\ Ewha Womans University, Seoul, Korea \\ \{smsong, kimmj, clrara, tlseo\} @ewhain.net \\ ${ }^{2}$ Korea Institute of Radiological and Medical Sciences, Seoul, Korea \\ $\{$ kmkim, larry\}@kcch.re.kr \\ ${ }^{3}$ Center for Computer Graphics and Virtual Reality \\ Ewha Womans University, Seoul, Korea \\ mhkimeewha.ac.kr
}

\begin{abstract}
Non-invasive imaging of small animal and its quantification techniques are needed to be evaluated by comparison with ex vivo image. To overcome the existing method, hand-operated comparison with the unaided eye observation, we present an algorithm that matches the target area in PET scanned in vivo with an autoradiography image acquired ex vivo. We applied a coregistration algorithm that uses voxel similarity to find the corresponding slices and to make quantitative measurements. Automatic matching of in vivo and ex vivo images is novel, and can provide better validation than manual matching techniques.
\end{abstract}

Keywords: Image registration, in vivo-ex vivo validation, small animal image.

\section{Introduction}

Small experimental animals such as mice and rats play a crucial role in enhancing our understanding of human disease. In vivo animal imaging technologies enable the development of a disease in its early stage, or the efficacy of a drug to be observed. These technologies allow repetitive, long-term follow-up studies and replace invasive and destructive observations by biopsy or histology. Many methods have been proposed for non-invasive extraction of quantitative information about a target area, and they can achieve better results than measurement using a caliper. Nevertheless, image-based analysis can be inaccurate. So in existing preclinical experiments the animal is sometimes sacrificed after non-invasive image analysis to obtain physical measurements in vitro or ex vivo for comparison. In comparing two images, it is difficult to find the same region of interest in both, and this is usually done manually until now. Besides, a quantitative comparison between the images may also be performed with the unaided eye[1,2] so the evaluation cannot be achieved objectively.

\footnotetext{
${ }^{*}$ Corresponding author.
} 
To overcome this approach, we propose an algorithm that automatically finds the same target area and extract its quantitative characteristics both in in vivo and ex vivo images using image registration. We also estimate the error range to establish the robustness of this approach to image quantification.

Nevertheless of necessity of in vivo - ex vivo image matching, existing image registration algorithms applied to small animal images have been designed to compare only between in vivo images so far. Furthermore, most of their methods are based on hardware or stereotactic approaches. Jan[3] proposed a way of combining PET, CT, and SPECT images using a calibration phantom and an animal holder, and Chow[4] aligned coordinate systems between images by moving the imaging cylinder. Rowland[5] and Shen[6] developed respectively, MRI-PET, MRI-SPECT image registration algorithms to observe structural and functional information simultaneously. In this paper we suggest the scheme of finding corresponding positions between in vivo - ex vivo images using an automatic and robust registration.

In Section 2 we described the features of the in vivo and ex vivo images used in this study, and the intensity-based image matching method used for automatic interimage comparison. Section 3 explains how we evaluated the matching method by quantifying the overlapped images. Experimental results and conclusions follow in Sections 4 and 5.

\section{Coregistration of in vivo and ex vivo Images}

In this section, we introduce two input image modalities: PET (Positron Emission Tomography) image which is acquired in vivo and autoradiography, which is obtained ex vivo. And we present an inter-image matching algorithm that performs registration based on voxel intensity statistics.

\subsection{Image Characteristics and Preprocessing}

PET provides a dynamic view of functional information about living animals, enabling the detection of biological and biochemical changes at the molecular level, using a radiotracer as a marker for a specific physiological function. In the resulting images we can see the distribution of the marker from the intensity of the radiation. The resulting data can quantify the distribution, and kinetics of a drug, and its likely mode of action on body function such as metabolism and blood flow. However, because of the low resolution and blurring artifact of PET images, it is difficult to determine the exact shape of a target area.

Autoradiography also involves injecting a radioactive tracer, but in this case the animal is then sacrificed to get more detailed anatomical information. The animal's organs are then sliced up and each slice is imaged with a detector that is sensitive to radiation.

Even though both PET and autoradiography use a radiotracer to visualize metabolic functions, autoradiography image shows fine anatomical structures because the sliced specimen is laid in contact with the radiation detector. To obtain autoradiography images after taking PET images of the small animal requires a preparation procedure that includes sacrificing, freezing and sectioning. During this 
time the radio-isotope used will decay, so we have to choose a radio-tracer with a long half-life so that the radioactivity remains strong until image acquisition is completed.

The image acquisition protocol is described in Fig. 1.

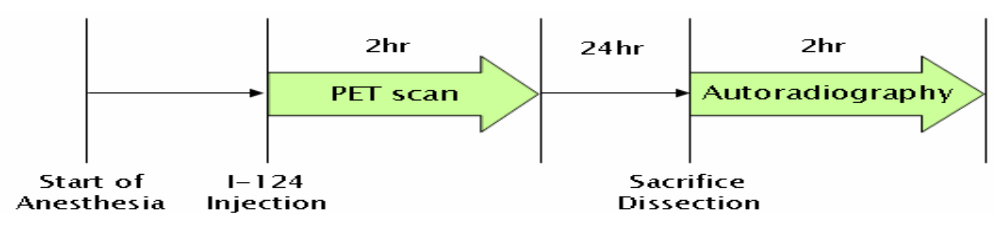

Fig. 1. Imaging protocol

However, much care is taken, the images acquired will still have some drawbacks. The slice thickness for autoradiography is $30 \sim 50 \mu \mathrm{m}$. This is so thin that some data can be lost during the slicing process (Fig. 2(a)), which can lead to inconsistency with PET data, and incorrect registration. Therefore we specify a small ROI (region of interest) as a boundary box at a preprocessing step (Fig. 2(c)). Only this region is considered during the subsequent image registration procedure, which speeds up the algorithm and makes it robust.

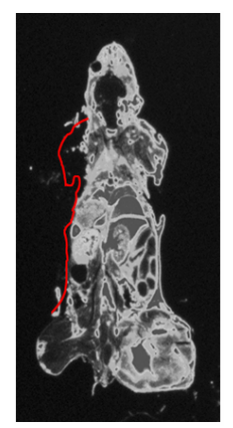

(a)

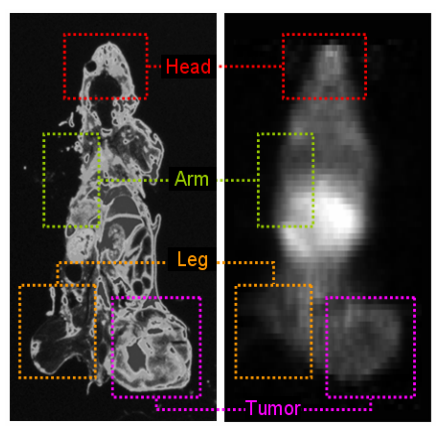

(b)

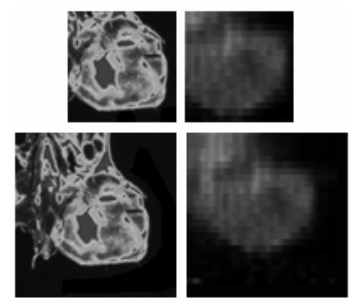

(c)

Fig. 2. Resolving problems with input images: (a) partial data loss, (b) inconsistency between PET and autoradiography data, (c) ROI(tumor area)

\subsection{Registration Using Voxel Similarity}

To compare a target area in PET and autoradiography data, it is necessary to match the same position in both images and then overlay them. PET is scanned for animal whole body three dimensionally at one time, but autoradiography is obtained slice by slice fashion. Because of the difficulties of slicing with uniform thickness, wholebody imaging by autoradiography is practically impossible. So when one autoradiography image is acquired during the experiment, finding corresponding image slice which shows the same tissue in PET volume data is needed. This matching processing is done by image registration. 


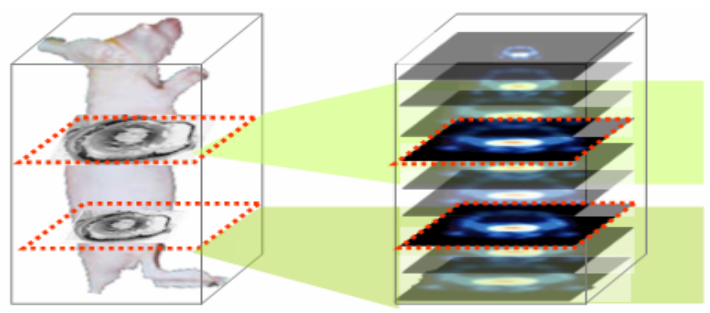

Fig. 3. Concept of finding corresponding slices in PET and autoradiography images

Image registration is the process of aligning images in order to correspond with their features[7]. In this process, one input image should be defined as a reference volume and the other is a test volume. And the test volume is repeatedly transformed until the most similar image is found.

In this paper, autoradiography image is treated as a reference because it has to be used as a validation standard and furthermore, when an image with higher resolution is used as a reference, image processing time and artifact due to the voxel interpolation can be reduced[8]. And to determine similarity between two images, we used an intensity based approach which does not need to extract feature points such as points or surfaces because it is difficult to segment geometrical structures in blurry PET image. In intensity based method the transformation factor can be computed by maximizing a similarity measure assuming statistical dependency between the intensity value of the two images.

In this study, we used mutual information[9], one of the most widely used measure in multi-modal image registration. Mutual information stems from information theory and statistics and it is expressed as the difference between the sum of the entropies of the individual images, $\mathrm{H}(\mathrm{A})$ and $\mathrm{H}(\mathrm{B})$, and the joint entropy of the combined image where they overlap, H(A,B) [10]. The entropy of a single image can be computed from the probability distribution of its intensities, and the joint entropy of two images can be estimated by computing a joint histogram of paired intensities. So this is a measure of the extent to which one image explains the other.

$$
I(A, B)=\frac{H(A)+H(B)}{H(A, B)} .
$$

However, mutual information measure is sensitive to the changes of the number of overlapped voxels and changes in overlap of very low intensity regions such as noise in the background. To overcome these kind of problems modified version by normalization has suggested and has proved very robust[11].

During the process of image registration two images A and B can should be corresponded by searching for an optimal transformation $\mathrm{T}$ which has the maximum normalized mutual information. Finding the transformation factor is represented as Eq. (2) where the original image $A(x)$ and the transformed image $B(T(x))$, where $x$ is a voxel in image $A$, and the closest voxel in image $B$ is $T(x)$, so that

$$
\hat{T}=\arg \max _{T} I(A(x), B(T(x)) .
$$


At this point, this requires the specification of the degrees of freedom of transformation. We assumed the animal body is fixed which means that there're no movements or structural changes to the animal during the whole image acquisition. Because the animal stays motionless because of gas anaesthesia during PET scanning and the animal is immediately sacrificed after PET acquisition and slicing for autoradiography is started as soon as cadaveric stiffening is apparent. Therefore we only applied the rigid transformation.

\section{Quantitative Measurement and Its Validation}

To validate the accuracy of image-based analysis, comparing the quantitative information by measuring the size, length, or biological activity of target tissue is required. Therefore, once the corresponding image slices have been found, we delineate the target area and extract its characteristics quantitatively from both in vivo and ex vivo image. In this section, we describe the target area detection algorithm and the methods of quantitative measurement used to obtain both morphological and biological information, shown in Fig. 4.

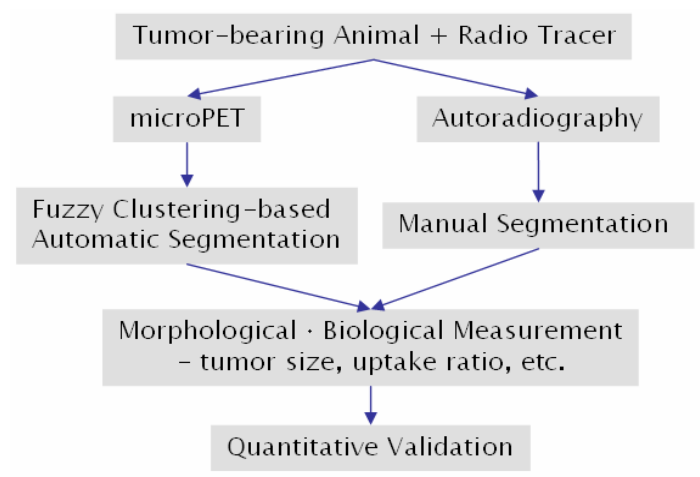

Fig. 4. Quantification of the target area

\subsection{Target Area Detection}

In this study, two image scans are acquired from a tumor-bearing mouse to observe metabolic activity in the tumor tissue and its growth rate. We segment the tumor area manually in the autoradiography data, because it is used as the reference standard in this study, as this data obtained ex vivo and shows the anatomical structure in fine resolution.

We now present a target area delineation algorithm to detect the tumor area in the PET data automatically [12] in two steps using a clustering algorithm. The tumor tissue looks bright compared to the surrounding normal tissue because of its high glucose uptake. We therefore can identify tumor tissue and other several types of normal tissue using voxel intensity. 
In the first step, we use an iterative thresholding method to separate the foreground, which is the whole of the body area, from the background in a very noisy PET image. Then, we calculate a membership function for each element in the foreground using the fuzzy C-means(FCM) objective function:

$$
J(U, V)=\sum_{j=1}^{c} \sum_{i}^{n}\left[u_{j}\left(x_{i}\right)\right]^{b} \cdot\left\|x_{i}-m_{j}\right\|,
$$

Under the condition

$$
\sum_{j=0}^{c} u_{j}=1, \sum_{j=0}^{c} u_{j}>0, u_{j} \in[0,1],
$$

Where $U$ is the matrix that contains the membership value of pixel $x_{i}$ with respect to each cluster, $u_{i}$ is an element of that matrix, and $m_{j}$ is the center of class $j$. Voxel clustering is done by minimizing this equation using the following iteration:

$$
m_{j}=\frac{\sum_{i=1}^{n}\left[u_{j}\left(x_{i}\right)\right]^{b} \cdot x_{i}}{\sum_{i=1}^{n}\left[u_{j}\left(x_{i}\right)\right]^{b}},
$$

$$
u_{j}\left(x_{i}\right)=\frac{\left(\frac{1}{\left\|x_{i}-m_{j}\right\|^{2}}\right)^{\frac{1}{(b-1)}}}{\sum_{k=1}^{c}\left(\frac{1}{\left\|x_{i}-m_{j}\right\|}\right)^{\frac{1}{(b-1)}}} .
$$

However, after intensity-based clustering, the tumor cluster may still include regions corresponding to normal organs such as the heart or bladder, which also show high intensity value because of glucose metabolism. To separate the tumor from this false positive region, we use a geometric clustering algorithm in which connected voxels are regrouped into the same cluster.

In Fig. 5(a) shows an image segmented into three clusters using brightness; the second cluster(shown in blue) is then divided into two using connectivity information. The small pink region is finally selected as the target area.
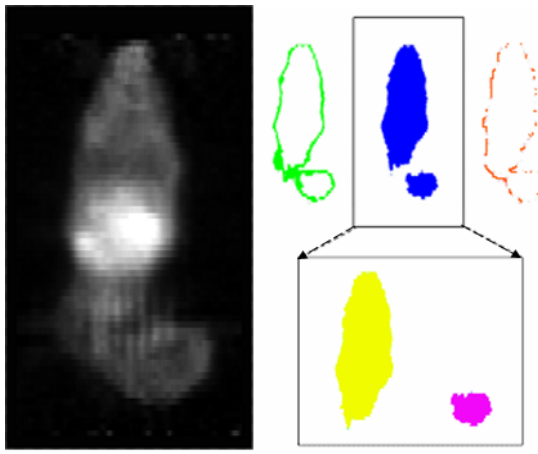

(a)

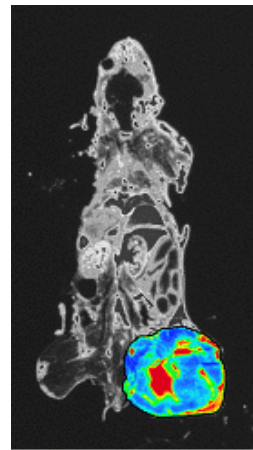

(b)

Fig. 5. Target area segmentation: (a) segmentation of PET data by fuzzy clustering, and (b) manual segmentation of autoradiography data 


\subsection{Measurement of Morphological and Biological Information}

Morphological information can be used to estimate the state of tumor growth, regression and regrowth. We compute the tumor size by counting the number of voxels in target area and multiplying by the voxel size in millimeters. To determine the length of the boundary of the tumor, we use a chain code algorithm to extract a contour and then count voxels along the line. We calculate the eigenvectors of the contour and consider its first principal axis as a diameter.

We can also extract biological information by computing metabolic activity, as a standard uptake value(SUV), in the tumor tissue:

$$
S U V=\text { meanROIactivity }\left(m C_{i} / c c\right) /\left(\text { injectedDose }\left(m C_{i}\right) / \operatorname{bodyWeight}(g)\right)
$$

This functional gives the information about tumor viability or a metabolic disorder. It can allow the stage of cancer to be determined, whether it is malignant or benign, and the efficacy of an anti-cancer drug observed.

\section{Implementation and Experimental Results}

We used PET and autoradiography to measure a tumor in a mouse. Hummm Burkitt Lymphoma Raji cell was injected into the right thigh of C57BR/cdJ SCID mouse and I-124 was injected into a vein as a biotracer. The spatial resolution of the PET scan was $256 \times 256 \times 63$ and the voxel size was $0.85 \times 0.85 \times 1.21 \mathrm{~mm}$ The resolution of each autoradiography slice was 1050x1998 and the voxel size was 40 $\mu \mathrm{m}$ To match PET and autoradiography data using image registration algorithm, every image slice of PET volume was compared to find the one which has the most similar intensity distribution with an autoradiography image.

Fig. 6 (a), (b) are the ROI of each input image in coronal view that is used in registration process. On this region, normalized mutual information is calculated and the plot of voxel similarity for a series of PET image slices is shown in Fig. 6 (d). The image which has the maximum value has to be transformed and Fig. 6 (c) shows its result. Table 1. provides quantitative comparisons for a selection of image pairs. It shows that, as the voxel similarity increases, the differences in quantitative parameters are reduced.

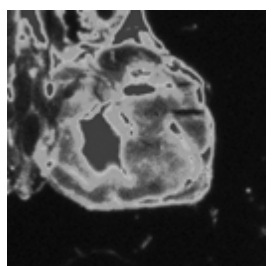

(a)

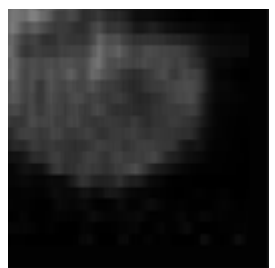

(b)

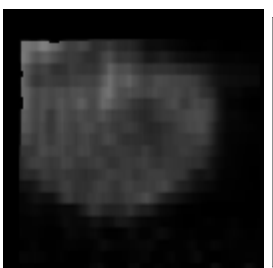

(c)

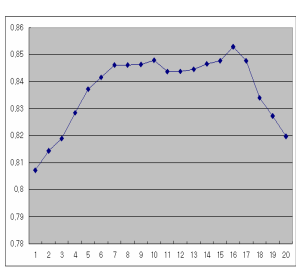

(d)

Fig. 6. PET-autoradiography Coregistration using voxel similarity: autoradiography, (b) PET, (c) registration result, (d) similarity measure 
Table 1. Quantitative comparison of target area characteristics

\begin{tabular}{|c|c|c|c|c|}
\hline & $\begin{array}{c}\text { Auto- } \\
\text { radiography }\end{array}$ & $\begin{array}{c}\text { PET } \\
\left(33^{\text {th }} \text { image }\right)\end{array}$ & $\begin{array}{c}\text { PET } \\
\left(37^{\text {th }} \text { image }\right)\end{array}$ & $\begin{array}{c}\text { PET } \\
\left(40^{\text {th }} \text { image }\right)\end{array}$ \\
\hline $\begin{array}{c}\text { Similarity } \\
\text { measure }\end{array}$ & $\cdot$ & 1.4864 & 1.5949 & 1.5827 \\
\hline Radius $(\mathrm{mm})$ & 0.85 & 0.83 & 0.85 & 0.86 \\
\hline Length $(\mathrm{mm})$ & 4.5 & 4.3 & 4.6 & 4.5 \\
\hline Size $\left(\mathrm{mm}^{3}\right)$ & 6529.23 & 6246.07 & 6624.60 & 6701.91 \\
\hline SUV & 10.04 & 9.7 & 9.8 & 11.20 \\
\hline
\end{tabular}

Because we consider the animal fixed during the image acquisition procedure, the registration error within any specific region of the mouse can be expected to be the same as the calculated for the global registration error. Therefore, the transformation parameters calculated for the small ROI can be applied to the entire image. After we have done this, we overlaid three images of the animal section changing the transparency to provide an intuitive understanding (Fig. 7).
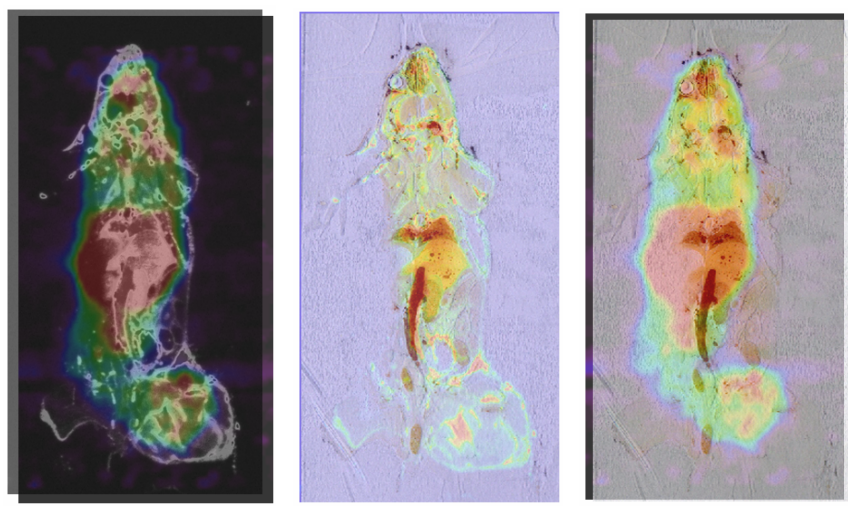

Fig. 7. PET, autoradiography, and visible-light images of a single section of the tumor-bearing mouse

\section{Conclusions and Future Work}

We have developed a coregistration algorithm to match in vivo PET and ex vivo autoradiography data. This allows image processing algorithms, including target area detection and quantification, applied to in vivo images to be validated by comparison with an ex vivo image. This comparison shows an error-rate within $0.1 \sim 0.2 \%$, which suggests that the proposed algorithm for in vivo quantification is sufficiently accurate. Results are proven robust even when the experimental image, especially autoradiography, has partial data missing problem which is frequently occurred during the image acquisition.

In the future, we plan to develop inter-image matching algorithms to compare autoradiography with other in vivo image modalities. 


\section{Acknowledgements}

This work is financially supported by the Korea Institute of Science \& Technology Evaluation and Planning (KISTEP) under the Real Time Molecular Imaging program and by the Ministry of Education and Human Resources Development (MOE), the Ministry of Commerce, Industry and Energy (MOCIE) and the Ministry of Labor (MOLAB) through the fostering project of the Lab of Excellency.

\section{References}

1. Chen, X., Park, R., Hou, Y., Tohme, M., Shahinian, A.H., Bading, J.R., Conti, P.S.: microPET and Autoradiographic imaging of GRP Receptor Expression with 64Cu-DOTA[Lys3]Bombesin in Human Prostate Adenocarcinoma Xenografts. The Journal of Nuclear Medicine 45(8) (2004)

2. Chang, C.H., Fan, K.H., Chen, T.J., Hsu, W.C., Jan, M.L., Tsai, T.H., Kao, P.F., Chen, C.F., Fu, Y.K., Lee, T.W.: Dynamic Evaluation of 18F-FDG Uptake by MicroPET and Whole-Body Autoradiography in a Fibrosarcoma-bearing Mouse Model. Journal of Formosan medical Association 103(11) (2004)

3. Jan, M.L., Chuang, K.S., Chen, G.W., Ni, Y.C., Chen, S., Chang, C.H., Wu, J., Lee, T.W., $\mathrm{Fu}$, Y.K.: A three-Dimensional Registration Method for Automated Fusion of micro PETCT-SPECT Whole-Body Images. IEEE Trans. on Med. Img. 24(7) (2005)

4. Chow, P.L., Stout, D.B., Komisopoulou, E., Chatziioannou, A.F.: A Method of Image Registration for Animal, Multi-modality Imaging. Physics in Medicine and Biology 51, 379-390 (2006)

5. Rowland, D.J., Garbow, J.R., Laforest, R., Snyder, A.Z.: Registration of 18f-FDG microPET and small-animal MRI. Nuclear medicine and Biology 32, 567-572 (2006)

6. Shen, D., Liu, D., Cao, Z., Acton, P.D., Zhou, R.: Coregistration of Magnetic Resonance and Single Photon Emission Computed Tomography Images for Noninvasive Localization of Stem Cells Grafted in the Infarcted Rat Myocardium. Mol. Imaging Biol. 9, 24-31 (2007)

7. Hajnal, Hill, Hawkes: Medical Image Registration. CRC Press (2001)

8. Pluim, J.P., Maintz, J.B., Viergever, M.A.: Interpolation artifacts in mutual informationbased image registration. Computer Vision and Image Understanding 77(2), 211-232 (2000)

9. Woods, R.P., Mazziotta, J.C., Cherry, S.R.: MRI-PET registration with automated algorithm. Journal of Computer Assisted Tomography 17(4), 536-546 (1993)

10. Pluim, J.P., Maintz, J.B., Viergever, M.A.: Mutual Information based Registration of Medical Images: a survey. IEEE Trans. On Medical Imaging 22, 986-1004 (2003)

11. Studholme, C., Hill, D.L.G., Hawkes, D.J.: An overlap Invariant Entropy Measure of 3D Medical Image Alignment. Pattern Recognition. 32, 71-86 (1999)

12. Lee, J., Song, S., Kim, K.M., Kim, M.-H.: Tumor detection from small animal PET using clustering method based on intensity and connectivity, World Congress on Medical Physics and Biomedical Engineering (2006) 